\title{
INFORME BIBLIOGRÁFICO NIETZSCHE Y EL LENGUAJE
}

La bibliografía sobre Nietzsche y el lenguaje es numerosa en sus distintos aspectos, ya que partimos de que Nietzsche como filólogo no deja de pensar en el lenguaje. En la bibliografía se recogen las perspectivas desde las que se estudia el lenguaje en la obra de Nietzsche: desde la metafísica, retórica, lingüística, semiótica, filosofía del lenguaje, etc. Dentro del elenco de obras citadas, se recogen también las referencias de algunas tesis doctorales, que por su interés, han acaparado la atención de los investigadores.

Para una mayor información sobre el tema del lenguaje en la obra y el pensamiento de F. Nietzsche, me remito a la base de datos, la Weimarer Nietzsche-Bibliographie, elaborada bajo la dirección de Erdmann Wilamovitz-Möllendorf y Josef Simon, entre otros, y que recientemente ha sido editada en cinco volúmenes por la editorial Metzeler, de Stuttgart. Para este tema véase especialmente el vol. 3, Sekundärliteratur Fortsetzung. Nietzsches geistige und geschichtlich-kulturelle Lebensbeziehungen, sein Denken und Schaffen, J. B. Metzler, Sttutgart/Weimar, 2000-2002 (www.metzlerverlag.de). Un informe sobre la citada bibliografía elaborado por Erdmann Wilamowitz-Möllendorf se puede encontrar en el número 2 de Estudios Nietzsche (249-258), donde se hace también una reseña sobre los volúmenes publicados.

AHREND, Thomas, «Das Verhältnis von Musik und sprache bei Nietzsche», NietzscheForschung, 2, 1995, 153-166.

ALBRECHT, Jörn, «Friedrich Nietzsche und das 'sprachliche Relativitätsprinzip'» Nietzsche-Studien, 8, 1979, 225-244.

BEHLER, Ernst, «Selbstkritik der Philosophie in der dekonstruktiven Nietzschelektüre», en Abel, Günter y Salaquarda, Jörg (ed), Krisis der Metaphysik, Walter de Gruyter, Berlin, 1989, pp. 283-306.

__, «Die sprachtheorie des frühen Nietsche», en T. Borsche, F. Gerratana y A. Venturelli (eds), op. cit., 99-111.

__, «Nietzsches Sprachtheorie und die literarische Übersetzung als Kunst», en Stadler, Ulrich (ed), Zwiesprache: Beiträge zur Theorie und Geschichte des Übersetzens, Stuttgart-Weimar, 1996, pp. 64-76.

__, «Nietzsches Sprachtheorie und der Aussagecharakter seiner Schriften», NietzscheStudien, 25, 1996, 64-86.

__, Ironie und literarische Moderne, Schöningh, München, 1997, «Nietzsche und das Spiel der Masken», pp. 250-278.

__, «Friedrich Nietzsche et la philosophie du langage du romantisme d'Iéna», Philosophie, 27, 1990, 57-75.

__, «Nietzsche's Study Greek Rhetoric», Research in Philosophy, 25, 1995, 3-26. 
BERGER, Hannelore, Untersuchungen zur Philosophie Nietzsches als einem Problem der Sprache, Univ. München (tesis dosctoral), 1969.

BIEBUYCK, Benjamin, «Eine Gleichniss- und Zeichensprache, mit der sich vieles verschweigen lässt. Figurations- und Metapherntheorie des späten Nietzsche», en Duhamel, Roland y Oger, Erik (eds), Die Kunst der Sprache und die Sprache der Kunst, Königshausen \& Neumann, Würzburg, 1994.

BIERLE, A. y CALDER III, W., «F. Nietzsche "Abriss der Geschichte der Beredsamkeit"», Nietzsche-Studien, 21, 1992, 363-389.

BISER, E, «Das Desiderat einer Nietzsche-Hermeneutik. Der Gang der Wirkungsgechichte», Nietzsche-Studien, 9, 1980, 1-37.

BLONDEL, E., «Nietzsche's style of affirmation: the metaphors of genealogy», en Yovel, Yirmiyahu (ed), Nietzsche as affirmative thinker, Martinus Nijhoff, Dordrecht, 1986, pp. 132-146.

__, «Vom Nutzen und Nachteil der Sprache für das Verháltnis Nietzsches. Nietzsche und der französische Strukturalismus», Nietzsche-Studien, 10/11, 1981-1982, 518-537.

BOHRER, Karl Heinz, Ästhetik und Rhetorik. Lecturen zu Paul de Man, Suhrkamp, Frankfurt a. M., 1993.

BÖNING, T., Metaphysik, Kunst, Sprache beim frühen Nietzsche, Walter de Gruyter, Berlín, 1988.

BORMANN, F., «Zur Chronologie und zum Text der Aufzeichnungen von Nietzsches Rhetorikvorlesungen», Nietzsche-Studien, 26 ,1997, 490-500.

BORSCHE, Tilman, GERRATANA, Federico y VENTURELI, Aldo (eds), "CentaurenGeburten». Wissenschaft, Kunst und Philosophie beim jungen Nietzsche, Walter de Gruyter, Berlín, 1994.

—, «Natur-Sprache: Herder - Humboldt - Nietzsche», en T. Borsche, F. Gerratana y A. Venturelli, op. cit.

BOWIE, Andrew, Aesthetics and subjectivity: from Kant to Nietzsche, Manchester University Press, 1993 (tr. Eleanor Leonetti, Visor, Madrid, 1999).

BREAZEALE, J. Daniel, «The word, the world, and Nietzsche», Philosophical forum, 6, 1974-1975, 301-320.

BROC-LAPEYRE, M, «La métaphore chez Nietzsche», en La métaphore: actes du colloque du 14/15 october 1987, Université de Sciences Sociales de Grenoble, Grenoble, 1988, pp. 138-154.

CADUFF, Corina, «Vom ,Urgrund' zum Supplement: Musik in den Sprachtheorien von Rousseau, Nietzsche und Kristeva», Musik \& Ästhetik, 1, 1997, 37-54.

CICORA, Mary A., «From metonymy to metaphor: Wagner and Nietzsche on language», German life and letters, 42, 1988-1989 (Oxford), 16-31.

CONWAY, D., «Beyond realism: Nietzsche's new infinite», International Studies in Philosophy, 22, 1990, 93-109.

COX, Christoph, Nietzsche. Naturalism and Interpretation, University of California Press, Berkeley, 1999.

CRAWFORD, Claudia, The Beginning of Nietzsche's Theory of Language, Walter de Gruyter, Berlín, 1988.

CREPON, Michel, Le malin génie des langues: Nietzsche, Heidegger et Rosenzweig, Vrin, París, 2000. 
DANTO, Arthur C., «Nietzsche und der semantische Nihilismus / aus dem Amerikan», en Guzzoni, Alfredo (ed.), 90 Jahre philosophische Nietzsche-Rezeption, Königstein/Ts., 1979, pp. 140-154.

DÁVILA DEL VALLE, Oscar G., «Sujeto, pensamiento, lenguaje: itinerario de una seducción», en Kerkhoff, Manfred H. (ed), Filosofía del desencanto: Nietzsche en Puerto Rico, San Juan, 1998, pp. 51-64.

DE MAN, Paul, Alegorías de la lectura. Lenguaje figurado en Rousseau, Nietzsche, Rilke y Proust, tr. de E. Lynch, Lumen, Barcelona, 1990.

DE SANTIAGO GUERVÓS, Luis Enrique, «Nietzsche y los límites del lenguaje: la fuerza del instinto», el mismo (ed.), La actualidad de Nietzsche, Contrastes, 1994 (suplemento ), 115-131.

__, Nietzsche y la polémica sobre «El nacimiento de la tragedia», Ágora, Málaga, 1994.

__, «El 'giro retórico' en la obra de Nietzsche», Er, Revista de Filosofía, 27, 2000, 11-34.

__, «El poder de la palabra: Nietzsche y la retórica», en F. Nietzsche, Escritos sobre retórica, Trotta, Madrid, 2000, pp. 9-80.

__, Arte y Poder. Aproximación a la estética de F. Nietzsche, Trotta, Madrid, 2004.

DIXSAUT, Monique, «Nietzsche et le métaphorique: des metáphores, des concepts et des catégories», La métaphore: actes du Colloque du 14/15 october 1987, Université de Sciences Sociales de Grenoble, Grenoble, 1988, pp. 155-172.

DJURIC, Mihailo, Nietzsche und die Metaphysik, Walter de Gruyter, Berlín, 1985.

DJURIC, Mihailo y SIMON, Josef (eds), Kunst und Wissenschaft bei Nietzsche, Königshausen \& Neumann, Würzburg, 1986.

__, Aktualität Nietzsches, 2 vols., Königshausen \& Neumann, Würzburg, 1984.

EBERSMEYER, Sabrina, «Philosophie als “Leidenschaft der Erkenntnis”: zur erkenntnistheoretischen Metaphorik in den Schriften Nietzsches», Nietzsche-Studien, 24, 1995, 17-44.

ELLRICH, Lutz, «Rhetorik und Metaphysik. Nietzsches "neue" ästhetische Schreibweise», en Nietzsche-Studien, 1994, 23, 241-272.

ENGEL, S. Morris, «An early Nietzsche fragment on language», Journal of the history of ideas, 24, 1963, 279-286.

FERRARIS, M., Nietzsche. Filologia, filosofia dell'arte, ermeneutica, ontologia, teoria della conoscenza.Laterza, Roma, 1998.

FIETZ, Rudolf, Medienphilosophie. Musik, Sprache und Schrift bei F. Nietzsche, Königshausen \& Neumann, Würzburg, 1992.

FIGL, J., «Nietzsche und die philosophische Hermeneutik des 20. Jahrhunderts: mit besonderer Berücksichtigung Diltheys, Heideggers und Gadamers», NietzscheStudien, 10/11, 1982, 408-430.

_- Interpretation als philosophisches Prinzip. Friedrich Nietzsches universale Tehorie der Auslegung im späten Nachlass, Walter de Gruyter, Berlín, 1982.

__ , «Hermeneutische Voraussetzungen der philologischen Kritik», Nietzsche-Studien, 13, 1984, 111-128.

FONTANA, Claudio, La scrittura della filosofia Platone, Vico, Nietzsche, Hestia, Cernusco, 1994.

FRAZIER, A.M., «Nietzsche on inspiration and language», Journal of thought, 9, 1974, 142-152. 
GASSER, Peter, Rhetorische Philosophie. Leseversuche zum metaphorischen Discurs in Nietzsches «Also sprach Zaratustra», Peter Lang, Berlín, 1992.

__, «"Columbus novus” _ Zum rhetorischen impetus von Nietzsches Philosophie», Nietzsche-Studien, 24, 1995, 137-161.

GEBHARD, Walter, «Zur Gleichnissprache Nietzsches. Probleme der Bildlichkeit und Wissenshcaftlichkeit», Nietzsche-Studien, 9, 1980, 61- 90.

- (ed.), Friedrich Nietzsche. Perspektivität und Tiefe, Peter Lang, Frankfurt a.M., 1982.

GILMAN, S. L. - BLAIR, C. - PARENT D.J (eds)., F. Nietzsche on Rhetoric and Language, Oxford University Press, Oxford, 1989.

GILMAN, Sander L., Nietzschean parody: An introduction to reading Nietzsche, Bouvier, Bonn, 1976.

GILMOUR, Douglas A., «On language, writing and the restoration of sight: Nietzsche's philosophical palinode», Philosophy and rhetoric, 27/3, 1994, 245-269.

GOTH, Joachim, Nietzsche und die Rhetorik, Niemeyer, Tübingen, 1970.

GRANIER, Jean, Le problème de la Vérité dans la philosophie de Nietzsche, Seuil, París, 1966.

GREMMLER, Claudia, Erkenntniskritik als ästhetisches Prinzip. Zum Verhältnis von Sprache und Kunst bei Nietzsche, Univesidad de Münster (tesis doctoral), 1984.

GRIMM, R.H., Nietzsche's theory of knowledge, Walter de Gruyter, Berlín, 1977.

HAAR, M., «Nietzsche and metaphysical language», Man and world, 4, 1971, 359-395.

__, «Nietzsche et la maladie du langage», Revue philosophique de la France et de l'étranger, 103/4, 1978, 403-417.

HALES, S. D., y WELSHON, R., Nietzsche's perspectivism, Univ. of Illionis Press, Chicago, 2000.

HAVAS, Randall, «Nietzsche and ordinary language philosophy», International studies in philosophy, 28, 1996. No. 3, 133-146.

HAZELTON, Roger, «Nietzsche's contribution to the theory of language», The philosophical review, 52, 1943, 47-60.

HEINEN, René, Sprachdynamik und Vernunft: Untersuchungen zum Spätwerk Nietzsches und Wittgensteins, Königshausen \& Neumann, Würzburg, 1998.

HENNIGFELD, Jochen, «Sprache als Weltansicht: Humboldt - Nietzsche - Whorf», Zeitschrift für philosophische Forschung, 30, 1976, 435-451.

HIDEO AKIYAMA, T., «Nietzsches Idee des 'grossen Stils'», Nietzsche-Studien, 3, 1974, 105-114.

HILL, Randolph Kevin, Language, mind and reality: Nietzsche's overcoming of metaphysics. Univ. of Illinois, Urbana-Champaign, 1992.

HINMAN, L. M., «Nietzsche, Metaphor, and Truth», Philosophy and Phenomenological Research, 43,1982, 179-199.

HÖDL, Hans Gerald, Nietzsches frühe Sprachkritik: Lektüren zu «Ueber Wahrheit und Lüge im aussermoralischen Sinne» (1873), WUV-Universitätsverlag, Viena, 1997.

HOFMANN, Johan Nepomuk, Wahrheit, Perspektive, Interpretation. Nietzsche und die philosophische Hermeneutik, Walter de Gruyter, Berlín, 1994.

IJSSELING, Samuel, Rhetoric and philosophy in conflict, Nijhoff, La Haya, 1976.

JIMENEZ MORENO, Luis, «Anotaciones sobre lenguaje y hermenéutica en Nietzsche», Revista de Filosofía, 8, 1985, 77-93. 
JORDAN, Lothar, «Nietzsche: Dekonstruktionist oder Konstruktivist», NietzscheStudien, 23, 1994, 226-240.

KAISER, Stefan, «Über Wahrheit und Klarheit», Nietzsche-Studien, 23, 1994, 6578.

KALB, Christof, «Symbolik des Leibes: Nietzsches frühe leib- und sprachphilosophische Überlegungen», Ars Semeiotic, 20, 1997, 275-299.

KAULHAUSEN, Marie Hed, Nietzsches Sprachstil: gedeutet aus seinem Lebensgefuihl und Weltverhältnis, Oldenbourg, München, 1977.

KIMOTO, Shin, «Nietzsche als Sprachlehrer: die Verfeinerung der Muttersprache zur Förderung der kritischen Denkweise», Doitsu Bungaku Ronshû = Die deutsche Literatur (Tokio), 28, 1995, 37-46.

KLEIN, Wayne, Between physiology and semiology: language and materiality in the writings of Nietzsche, New School for Social Research (tesis doctoral), New York, 1993.

KOFMAN, Sarah, Nietzsche et la scène philosophique, UGE, París, 1979

__, Nietzsche et la métaphore, Galilée, París, 1983.

KOPPERSCHMIDT, Josef - SCHANZE, Helmut (eds), Nietzsche oder “Die Sprache ist Rhetorik”, Fink, München, 1994.

KREMMER-MARIETTI, A., Thèmes et Sturctures dans l'Oeuvre de Nietzsche, Lettres Modernes, París, 1957.

__, Nietzsche et la rhétorique, PUF, París, 1991.

__, L'Homme et ses labyrinthes, Harmattan, París, 1999.

KÜNZLI, Rudolf E., «Nietzsche und die Semiologie», Nietzsche Studien, 5, 1976, 263-288.

KUSMER, Robert L., Language, poetry, and truth: A study of Nietzsche's views on language in its aesthetic and philosophical contexts, Northwestern Univ. (tesis doctoral), Chicago, 1983.

LACOUE-LABARTHE, Ph., «Le détour», Poetique, 5, 1971, 53-76.

LÉVESQUE, Claude, Dissonance: Nietzsche à la limite du langage, Éditions Hurtubise HMH, Lasalle, 1988.

LILLY, Reginald Storrs, The place of language: art and nihilism in Nietzsche and Heidegger, Duquesne Univ. (tesis doctoral), 1984, 237.

LINGIS, A., «Effets de langage», en Dominique Janicaud (ed), Nouvelles lectures de Nietzsche: à l'occasion du centième anniversaire de la composition à Nice du livre III d' «Ainsi parlait Zarathoustra», L'Âge D'Home, Lausanne, 1985, 50-57.

__, «The language of The gay science», en Tymieniecka, Anna-Teresa (ed), The philosophical reflection of man in literature: selected papers from several conferences held by the International Society for Phenomenology and Literature in Cambridge, Dordrecht (Analecta Husserliana 12), Massachusetts, 1982, 313-329.

LLINARES, J. B, Hombre, arte y lenguaje. Una investigación sobre el joven Nietzsche, Univ. de Valencia, Valencia, 1982.

__, «La filosofía del lenguaje en Nietzsche», en Metafísica y pensamiento actual. Conocer a Nietzsche, Sociedad Castellano-Leonesa de Filosofía, Salamanca, 1996, pp. 237-258.

__, «La filosofía del lenguaje en el Zaratustra de Nietzsche», Revista de Filosofía, Valencia, 2, 1984, 51-62. 
LONGO, Silvano, «Nietzsche e il palinsesto del linguaggio», Verifiche, 15, 1986 (Trento), 423-436.

LÖW, R., Nietzsche sophist und Erzieher. Philosophische Untersuchungen zum systematischen Ort von F. Nietzsches Denken, Acta Humaniora, Weinheim, 1984.

LUNGSTRUM, Janet, «Wittgenstein and Nietzsche: agonal relations in language», Deutsche Vierteljahrsschrift für Literaturwissenschaft und Geistesgeschichte, 69, 1995, 300-323.

LYNCH, E., Dioniso dormido sobre un tigre, Destino, Barcelona, 1993.

MAH, Harold, «The epistemology of the sentence: language, civility, and identity in France and Germany, Diderot to Nietzsche», Representations, 47, 1983 (Berkeley), 64-84.

MAKARUSHKA, Irena S. M, Religious imagination and language in Emerson and Nietzsche, St. Martin's Press, Houndmills, 1994.

MARTON, Scarlett, «Linguagem e consciência na obra de Nietzsche», en Favaretto, Celso (ed), Epistemologia das ciências sociais, Sêrie cadernos PUC, São Paulo, 1984, 125-135.

MASINI, Ferruccio, Lo scriba del caos. Interpretatione di Nietzsche, Il Mulino, Bolonia, 1978.

MEIJERS, A. y ESTINGELIN, M., «Konkordanz zu den wörtlichen Abschriften und Übernahmen von Beispielen und Zitaten aus Gustav Gerber: Die Sprache als Kunst (Bomberg, 1871) in Nietzsches Rhetorik Vorlesung und in "Über wahrheit und Lüge im aussermoralischen Sinne”», Nietzsche-Studien, 17, 1998, 356-368.

MEIJERS, A., «Gustav Berger und Friedrich Nietzsche. Zum historischen Hintergrund der prachpnilosophischen Auffassungen des frühen Nietzsche», Nietzsche-Studien, 17, 1988, 369-90.

MONTOYA, Jairo, «Lenguaje, genealogía e interpretación», en Montoya, Jairo (ed), Nietzsche: 150 años, Santiago de Cali (Medellín), 1995, 89-116.

MORENO MONTORO, Marcial, «La apuesta literaria de Nietzsche», Diálogos, 26, 1991, 89-100.

MOROWA, Hans, Sprache und Stil von Nietzsches «Zarathustra»: ein Beitrag zur Erkenntnis seines geistig-seelischen Ausdrucksgehalts, Schubert, München, 1958.

MOST, Glenn y FRIES, Thomas, «Die Quellen von Nietzsches Rhetorik-Vorlesung», en T. Borsche, F. Gerratana y A. Venturelli (ed), op. cit., pp. 17-46.

MÜLLER, Daniel, Wider die «Vernunft in der Sprache». Zum Verhältnis von Sprachkritik und Sprachpraxis im Schreiben Nietzsches, Gunter Narr, Tübingen, 1995.

MÜLLER, Thomas, Die Poetik der Philosophie: das Prinzip des Perspektivismus bei Nietzsche, Campus Verlag, Frankfurt, 1995.

MÜLLER-RICHTER, K. et al., Der Streit und die Metapher, Wissenschaftliche Buchgesellschaft, Darmstadt, 1998.

NATOLI, Salvatore, Ermeneutica e genealogia. Filosofia e metodo in Nietzsche, Heidegger, Foucault, Feltrinelli, Milán, 1981.

NAUMANN, Barbara, «Nietzsches Sprache 'Aus der Natur': Ansätze zu einer Sprachtheorie in den frühen Schriften und ihre metaphorische Einlösung in Also sprach Zarathustra», Nietzsche-Studien, 14, 1985, 126-163.

NIEHUES-PRÖBSTING. H., Überredung zur Einsicht, Suhrkamp, Frankfurt a. M., 1987. 
NIES, Karl-Dieter, Sprache und Moral: Untersuchungen zu Nietzsches Theorie von Genese und Entwicklung der Sprache, insbesondere im Zusammenhang seiner späten Moralphilosophie, P. Lang, Frankfurt, 1991.

NISHIO, Kanji, «Sprache und Ding: Nietzsches Sprachauffassung in Die Geburt der Tragödie» Doitsu Bungaku», Die deutsche Literatur, 37, 1966. 59-76.

OLIVER, Kelly Ann, “Woman's" language, man's voice: A reader of gender and language in Nietzsche, Northwestern Univ, Chicago, 1987.

ORSUCCI, Andrea, «Unbewusste, Anticipationen, Übertragungen. Über Nietzsches Verhältnis zu Karl Friedrich Zöllner und Gustav Gerber», en T. Borsche - F. Gerratana - A. Venturelli (ed), op. cit., pp. 208-216.

ORTEGA DEL BLANCO, Juan José, «La crítica del lenguaje en Nietzsche», Anales de filosofía, 3, 1985, 149-157.

OTTO, Detlef, Wendungen der Metapher: zur Übertragung in poetologischer, rhetorischer und erkenntnistheoretischer Hinsicht bei Aristoteles und Nietzsche, Fink, München, 1998.

__, «Die Version der Mefapher zwischen Musik und Begriff», en T. Borsche, F. Gerratana y A. Venturelli (ed), op. cit., pp. 167-192.

PAUTRAT, Bernard, Version du soleil. Figures et système de Nietzsche, Seuil, París, 1971.

PARMEGGIANI, Marco, Nietzsche: Crítica y proyecto desde el nihilismo, Ágora, Málaga, 2002, cap. IV: «Hermenéutica de la experiencia interior», pp. 83-100.

__, «Sujeto, pensamiento y lenguaje en Nietzsche», Contrastes, V, 2000, 263-283.

POENITSCH, Andreas, Bildung und Sprache zwischen Moderne und Postmoderne: Humboldt, Nietzsche, Ballauff, Lyotard, Die blaue Eule, Essen, 1992, cap. II: «Die Wieterführung und Verschärfung der Problematik in der Bildungstheorie des frühen Nietzsche - Nietzsches negative und positive Sprachtheorie», pp. 50-94.

PÖKSEN, Uwe, «Die Funkionen einer naturwisseshcatlichen metapher in einem Satz Nietzsches», Nietzsche-Studien, 13, 1984, 443-447.

POLIDORI, Fabio, Necessità di una illusione: lettura di Nietzsche, Guerini, Milano, 1995.

PORTER, Jame, «Nietzsche's Rhethoricc: Theory and Strategy», Philosophy and Rhetoric, 27, 1994, 218 ss.

PRADO, Plinio Walder Jr., «The concealed art of language: fragments of the young Nietzsche», Journal of Nietzsche studies, 1, 1991, 23-33.

PRATICÒ, Giovanni: «F. Nietzsche: verità e linguaggio come alienazione metafisica», en Ciaravolo, Pietro (ed), Nietzsche - Stirner, Roma, 1984, pp. 67-79.

REY, Jean-Michel, L'enjeu des signes. Lecture de Nietzsche, Seuil, París, 1971.

RODRIGUEZ, Mariano, «Nietzsche y el lenguaje», Revista de filosofía, 8, 1985, 63-76

ROVATTI, Pier Aldo, Como la luz tenue. Metáfora y saber, Gedisa, Barcelona, 1999.

RUPP, Gerhard, Rhetorische Strukturen und kommunikative Determinanz, Peter Lang, Frankfurt a. M., 1976.

SCHACHT, Richard, «Philosophy as linguistic analysis: a Nietzschean critique», Philosophical Studies, 25/1, 1974 (Boston), 153-171.

SCHEER, Brigitte, «Die Bedeutung der Sprache im Verhältnis von Kunst und Wissenschaft bei Nietzsche», en Djuric, M. y Simon, J. (ed), op. cit., 101-111. 
SCHLECHTA, Karl, «Nietzsche über den Glauben an die Grammatik», NietzscheStudien, 1, 1972, 353-358.

SCHLÜPMANN, Heide, Friedrich Nietzsches ästhetische Opposition: der Zusammenhang von Sprache, Natur und Kultur in seinen Schriften 1869-1876, Metzler, Stuttgart, 1977.

SCHMIDT, Siegfried J., Sprache und Denken als sprachphilosophisches Problem von Locke bis Wittgenstein, Nijhoff, La Haya, 1968.

SCHÖFFEL, Geor, Zur Logik sprachlicher Bilder, Westdeutscher Verlag, Optladen, 1987.

SCHRIFT, Alan D., Nietzsche and the question of interpretation. Routledge, Londres, 1900.

__- «Language, metaphor, rhetoric: Nietzsche's deconstruction of epistemology», Journal of the history of philosophy, 23/3, 1985, 371-395.

SHEPARD, Paul M., Language, truth and power in ancient Greek thought: prolegomena to Nietzsche, Univ. of Massachusetts (tesis doctoral), Amherst, 1993.

SIGNORI, Alberto, «Pensiero e linguaggio in F. Nietzsche», Nuova Antologia, 132/2203, 1997, 70-107.

SIMON, Josef, «Grammatik und Wahrheit. Über das Verhälnis Nietzsches zur spekulativen Satzgrammatik der methaphysischen Traditionen», Nietzsche-Studien, 1, 1972, 1-26.

__, «Sprache und Sprachkritik bei Nietzsche», en Lutz-Bachmann, Matthias (ed.), Über Friedrich Nietzsche: eine Einfuihrung in seine Philosophie, Frankfurt, 1985, 63-97.

SIMONIS, Annette, «Sprache und Denken: Sprachreflexion bei Karl Philipp Moritz und Friedrich Nietzsche», Zeitschrift für Literaturwissenschaft und Linguistik, 25, 1995, 124-133.

SINI, Carlo, Semiotica e filosofia: segno e linguaggio in Peirce, Nietzsche, Heidegger e Foucault, Il Mulino, Bologna, 1978.

SONDEREGGER, Robert, «Friedrich Nitezsche und die Sprache», Nietzsche Studien, 2, 1973, 1-30.

SONDEREGGER, Stefan, «Friedrich Nietzsche und die Sprache: eine sprachwissenschaftliche Skizze», Nietzsche-Studien, 2, 1973, 1-30.

SONODA, Muneto, «"Die Dichter lügen zu viel”: Nietzsche und die Sprache», Doitsu Bungaku = Die deutsche Literatur, 85, 1990 (Tokyo), 42-51.

STACK, George J., Lange und Nietzsche, Walter de Gruyter, Berlín, 1983.

STEGMAIER, Werner, «Nietzsches Zeichen», Nietzsche-Studien, 29, 2000, 41-69.

STERN, Joseph Peter, «Dasein als Metapher: zu Nietzsches Sprachphilosophie», Frankfurter Hefte, 31, 1976, 55-66.

STEUER, Daniel, «Mit der Stimme im Rücken: Nietzsche, Wittgenstein und die Sprache», German life and letters, 48/4, 1995, 499-515.

STINGELIN, Martin, «Nietzsches Wortspiel als Reflexion auf poet(olog)ische Verfahren», Nietzsche-Studien, 17, 1988, 336-349.

__, «Nietzsche, die Rhetorik, die decandence», Sprache und Literatur, 75-76, 1995, 27-44.

__, «Unsere ganze Philosophie ist Berichtigung des Sprachgebrauchs»: Friedrich Nietzsches Lichtenberg-Rezeption im Spannungsfeld zwischen Sprachkritik (Rhetorik) und historischer Kritik (Genealogie), Fink, München, 1996. 
__, «Die Rhetorik des Menschen», Nietzsche-Studien, 24,1995, 336-43.

STRATHMAN, Christopher Anthony, Ironic hermeneutics in Schlegel, Byron, Nietzsche and Joyce, Univ. of Notre Dame (tesis doctoral), Indiana, 1993.

TANESINI, Alessandra, "The 'spider's web' and the 'tool': Nietzsche vis-à-vis Rorty on metaphor», en Sedgwick, Peter R. (ed), Nietzsche: a critical reader, Oxford, Cambridge, 1995, pp. 276-293.

TEBARTZ-VAN ELST, Anne, Ästhetik der Metapher, K. Alber, München, 1994.

THALKEN, Michael, Ein bewegliches Heer von Metaphern: Sprachkritisches Sprechen bei Friedrich Nietzsche, Gustav Gerber, Fritz Mauthner und Karl Kraus, Peter Lang, Frankfurt, 1999.

THOMAS, Douglas, Reading Nietzsche Rhetorically, Guilford Press, New York, 1998.

THÜRING, Hubert, «Friedrich Nietzsches mnemotechnisches Gleichnis: von der 'Rhetorik' zur 'Genealogie'», en Kopperschmidt, J. y Schanze, H. (ed), op. cit., pp. 63-84.

THURNHER, R., «Sprache und Welt bei Friedrich Nietzsche», Nietzsche-Studien, 9, 1980, 38-60.

UNGEHEUR, G., «Nietzsche über Sprache und Sprechen, über Wahrheit und Traum», Nietzsche-Studien, 12, 1983, 134-213.

VOLLMER, Michael, Das gerechte Spiel: Sprache und Individualität bei Friedrich Nietzsche und Peter Handke, Königshausen \& Neumann, Würzburg 1995.

WILLEMSEN, Roger, «Dionysisches Sprechen: zur Theorie einer Sprache der Erregung bei Musil und Nietzsche», Deutsche Vierteljahrsschrift für Literaturwissenschaft und Geistesgeschichte, 60, 1986, 104-135.

WILLWOCK, J., «Die Reflexion der Rhetorik in der Philosophie Friedrich Nietzsches», Philosophisches Jahrbuch, 89,1982, 3-55.

ZUNJIC, S., «Begrifflichkeit und Metapher. Einige Bemerkungen zu Nietzsches Kritik der philosophischen Sprache», Nietzsche-Studien, 16, 1987, 149-163.

elaborado por Luis E. De Santiago Guervós Universidad de Málaga 